\title{
Irreversible Blindness due to Prolonged use of Hydroxychloroquine for Malaria Prophylaxis: A case Report
}

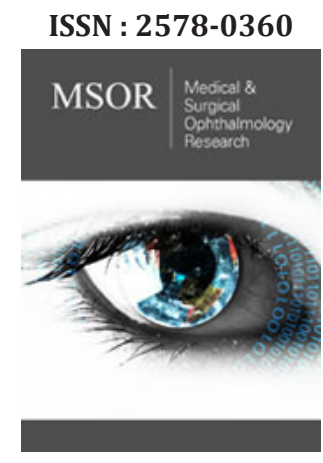

*Corresponding author: Ejike Egbu, Consultant Ophthalmologist, Lily Hospitals Limited,Warri, Nigeria

Submission: 酶 November 17, 2020

Published: 㓩 November 25, 2020

Volume 3 - Issue 1

How to cite this article: Ejike Egbu, Johnpaul Okolo. Irreversible Blindness due to Prolonged use of Hydroxychloroquine for Malaria Prophylaxis: A case Report. Med Surg Ophthal Res. 3(1). MSOR. 000553. 2020.

DOI: 10.31031/MSOR.2020.03.000553

Copyright@Ejike Egbu, This article is distributed under the terms of the Creative Commons Attribution 4.0 International License, which permits unrestricted use and redistribution provided that the original author and source are credited.

\author{
Ejike Egbu ${ }^{1 *}$ and Johnpaul Okolo ${ }^{2}$ \\ ${ }^{1}$ Consultant Ophthalmologist, Lily Hospitals Limited,Nigeria \\ ${ }^{2}$ Consultant Ophthalmologist, Tulsi Chanrai Foundation Eye Institute, Nigeria
}

\begin{abstract}
Chloroquine and hydroxychloroquine have been used over the years for the treatment of malaria and rheumatic diseases. It was also proposed for the treatment of COVID-19 in the solidarity and recovery clinical trials that have been suspended by the World Health Organisation (WHO). Malaria remains a leading cause of morbidity and mortality in endemic regions of West Africa. The desire to stay malaria-free can make some people indulge in chemoprophylaxis, using readily available over the counter medications such as chloroquine and hydroxychloroquine. The mechanism of retinal toxicity from these agents is thought to be due to their high affinity for melanin in the retinal pigment epithelium through the activity of OATP1A2, a polypeptide involved in the recycling of all trans-retinol. With the advent COVID-19, the use of hydroxychloquine may increase, predisposing more people to the harmful effects of these drugs. There is need to establish a baseline macular function before treatment with hydroxychloroquine, in line with the recommendations of the American Academy of Ophthalmology. This article reports the case of hydroxylchloroquine maculopathy after twenty years of chemoprophylaxis for malaria.
\end{abstract}

Keywords:Hydroxychloroquine; Malaria; Toxic maculopathy; Irreversible blindness; COVID-19; Bull's eye maculopathy

Abbreviations:HCQ: Hydroxychloroquine; SLE: Systemic Lupus Erythematosus; RPE: Retinal Pigment Epithelium; MFERG: Multifocal Electroretinogram; AAO: American Academy of Ophthalmology

\section{Introduction}

Hydroxychloroquine (HCQ) is a medication commonly used by rheumatologists for the treatment of Rheumatoid Arthritis (RA), immune disease, malaria, Systemic Lupus Erythematosus (SLE), dermatologic conditions and other connective tissue disorders with less toxicity compared to chloroquine [1,2]. Though it seldom occurs, hydroxychloroquine causes severe retinal toxicity that persists even after the cessation of the medication [3-5]. Patients at the early stages of toxicity do not really experience symptoms but few patients have paracentral scotoma on central visual field analysis. Advanced stages may lead to the loss of acuity, peripheral vision and poor night vision [3]. Other reported visual symptoms of hydroxychloroquine toxicity include dimness of vision, yellow flashes of light, decreased color vision, decreased dark adaptation, seeing green or red haloes, double vision and increased light sensitivity. Ocular findings on examination include cornea deposits, posterior subcapsular lens opacities, blunting of the foveal reflex and bull's eye maculopathy which is a classic finding. Retinal pigment irregularity, bone spicule formation, retinal vascular attenuation and optic disc pallor are also reported findings [6].

Retinal toxicity due to the use of hydroxychloroquine occurs when the cumulative dose exceeds $1000 \mathrm{~g}$ and a daily dose of $400 \mathrm{mg}$. Patients above 60 years of age or who have had prolonged consumption of at least 5 years of usage are at increased risk [1]. The mechanism of action of HCQ is multivalent and yet to be fully explained. However, studies have shown that the drug affects the metabolism of retinal cells and binds to melanin in the retinal pigment epithelium (RPE), which interferes with lysosomal activity. This alters the membrane stability and disrupts deoxyribonucleic acid (DNA) function by depressing protein synthesis 
and reducing cell replication and transcriptional activity $[7,8]$. This explains why its inhibitory effect on vision is progressive even after the cessation of the drug. The effect of chloroquine and hydroxychloroquine on the RPE is thought to be due to the inhibition of a polypeptide, 1A2 (OATP1A2) which is involved in the recycling of all-trans retinol in the phototransduction cascade [9]. In a publication on hydroxychloroquine retinopathy, the authors concluded that with long term use, some patients could develop severe sight threatening conditions and therefore advocated for a practical protocol for screening of patients as early as possible in line with the AAO guideline. The guideline recommends that at commencement of treatment with HCQ, there should be a detailed ocular examination to screen for any preexisting condition, a central 10-2 white-on-white central visual field assessment using a humphery visual field analyzer and a funduscopic examination using any one of the following modalities: Fundus Autofluorescence (FAF), Multifocal Electroretinogram (MFERG), or spectral domain OCT (SD-OCT) [3]. Michel Michaelides et al also found that retinal toxicity due chloroquine and hydroxychloroquine did not show any reversal after cessation of the drug in a clinical study of 16 patients that used hydroxychloroquine for an average of 13 years and concluded that there was need to educate physicians on the devastating consequences of using these agents over a long period of time [4].

Drug interaction with Tamoxifen, a licensed drug for breast cancer prevention has been shown to interfere with HCQ toxicity by concurrently increasing its risk and it is described as a 'major risk factor' in the recent revised American Academy of Ophthalmology (AAO) guidelines, 2016. Hence, it is advised to avoid the combination of these drugs to prevent higher toxicity [9]. More recently controversies over the toxicity of hydroxychloroquine was a huge concern in the solidarity and recovery clinical trials. Solidarity is a global clinical trial that tried to find suitable drug treatment for COVID-19 in which 12000 patients were enrolled from 500 hospitals in about 30 countries while recovery is a UK based randomized clinical trial that had 1500 patients out of a proposed 10680 participants. The two clinical trials were stopped by the WHO for lack of evidence supporting the use of hydroxychloroquine [10].

\section{Case Report}

A fifty-four-year-old man who presented on account of insidious painless reduction in vision for distance and near vision over the past one year. He experienced increased difficulty reading his books. This problem was worse with reduced illumination. There was no family history of blindness. He is not a smoker and does not have diabetes mellitus or hypertension. He volunteered a history of ingesting over-the-counter hydroxychloroquine tablets on a monthly basis for malaria prophylaxis for about twenty five years. There was also no history of chronic depression and had not been managed for any psychiatric condition. He is a lawyer and was preparing for a postgraduate degree examination at the time of presentation. On ocular examination, unaided visual acuity was $3 / 60$ in both eye and did not improve with refraction. His near visual acuity was N36 in both eyes. Slit lamp examination revealed normal findings in the anterior and posterior segments. Funduscopic examination with a 90D lens showed mottling of the fovea in both eyes. Optical coherence tomography revealed a disruption of the retinal pigment epithelial layers of the retina in both eyes, see (Figures 1-3). These findings suggested hydroxychloroquine maculopathy. He was reviewed for any psychiatric problems and was found to be normal. He got low vision aids, hand-held magnifiers and was advised to discontinue the use hydroxychloroquine and use other methods of malaria prophylaxis such as the use of mosquito net.

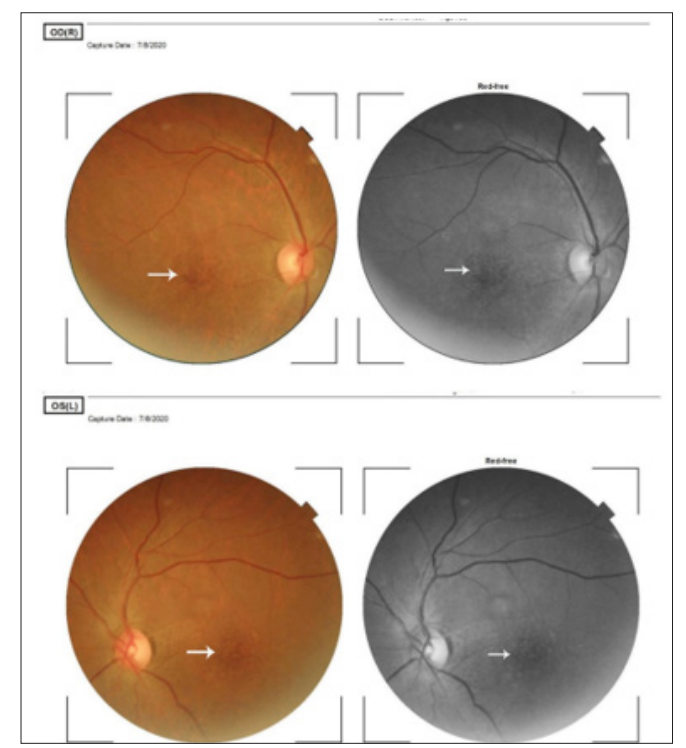

Figure 1: Panes 1A and 1B show the fundus photographs of the right and left eye respectively. The white arrows indicate mottling of the fovea due to hydroxychloroquine toxicity. This is typically described as bull's eye maculopathy. 


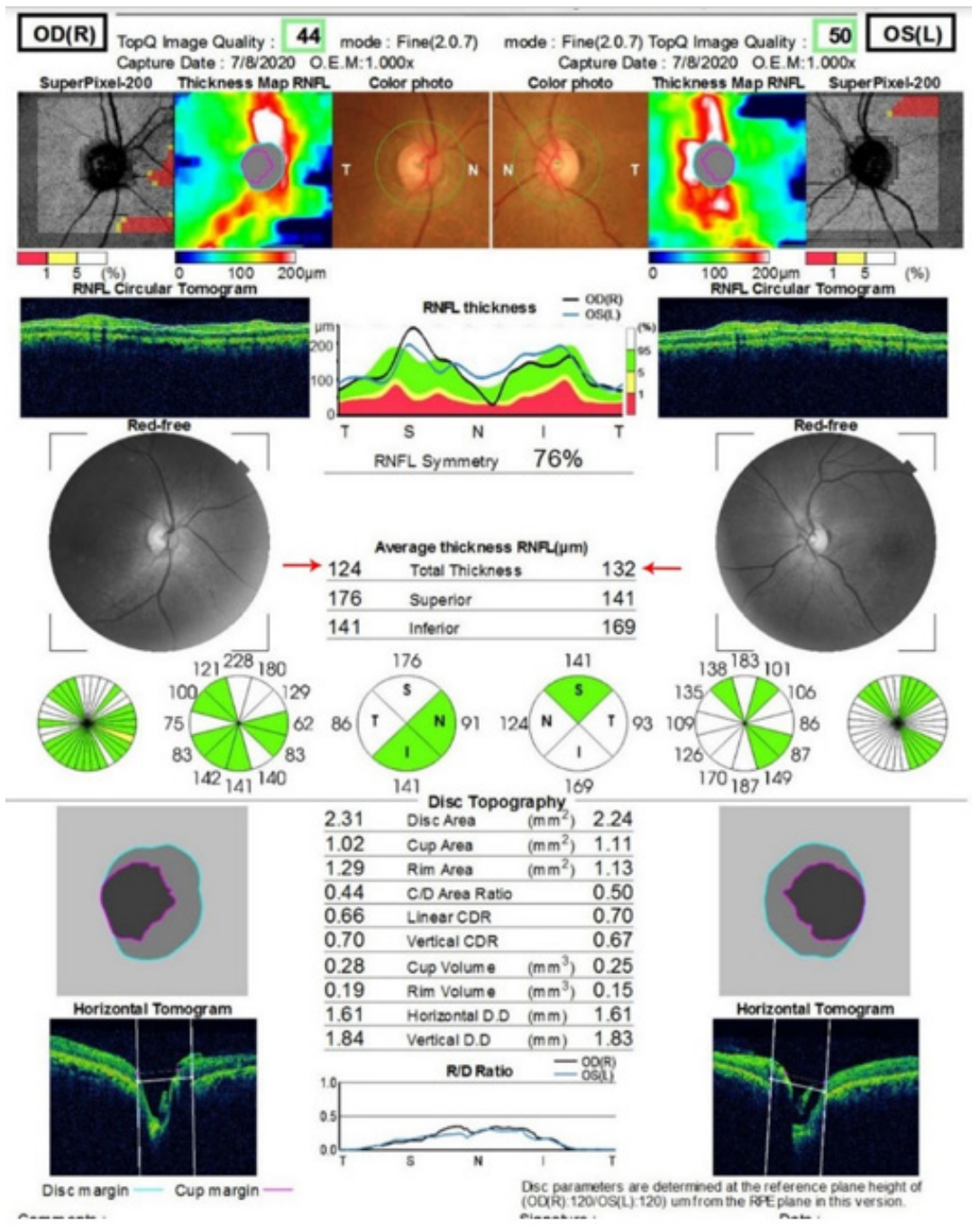

Figure 2: Show the optical coherence tomography of the optic discs of both eyes. The optic discs have normal thickness, see red arrows.

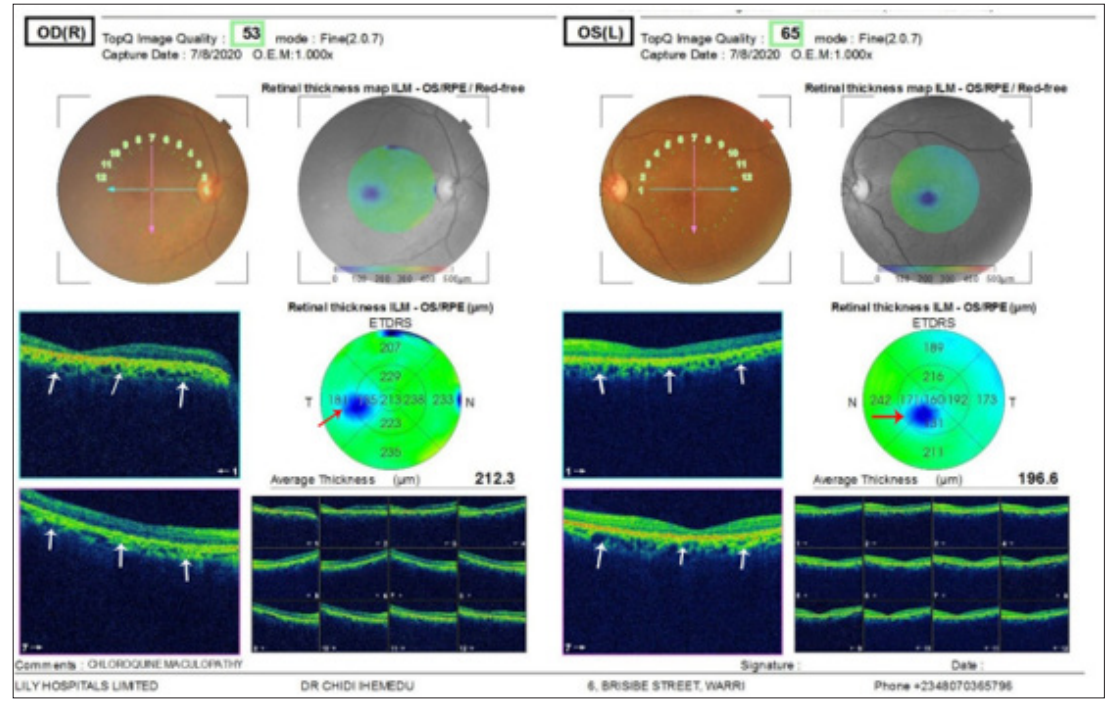

Figure 3: Panes 3A and 3B represent the optical coherence tomography scans of the right and left eye respectively. The white arrows show disruption of the retinal pigment epithelium typical of hydroxychloroquine maculopathy. 


\section{Discussion}

Hydroxychloroquine (HCQ) is beneficial in the treatment of several ailments such as malaria, Systemic Lupus Erythematosus (SLE) and other auto-immune diseases. The common side effects observed in acute toxicity include gastrointestinal disturbances such as dyspepsia, nausea and vomiting, headaches and visual disturbances. It has been found that excessive usage has led to serious visual problems and irreversible blindness [2]. These problems can occur in individuals who take this drug at a cumulative dose of $1000 \mathrm{~g}$ or constantly for a minimum of five years. Most of the articles on toxicity from antimalarial drugs focus on chloroquine as there are few reported cases of macular toxicity due to hydroxychloroquine for the treatment or prophylaxis of malaria. The index patient had constantly used hydroxychloroquine for the prophylaxis of malaria for over twenty years, buying the drugs from pharmacy shops without a valid prescription. One of the factors that may be implicated in the wrong use of hydroxylchloroquine for malaria prophylaxis by this patient could be due to the lack of clear guidelines for long term malaria chemoprophylaxis in endemic regions. The dosing of hydroxychloroquine for malaria is usually reported as base equivalent and as salt for the management of rheumatoid conditions. For malaria in adults, hydroxychloroquine is taken as an oral medication and its recommended prophylactic dose is $400 \mathrm{mg}$ salt (310 mg base) administered once a week. When traveling to an endemic region, the individual should take the drug for two weeks prior to exposure and up to four weeks after leaving the region. The therapeutic dose of hydroxychloroquine, an initial loading dose of $800 \mathrm{mg}$ salt (equivalent to $620 \mathrm{mg}$ base), followed by $400 \mathrm{mg}$ salt (310mg base) after six, twenty four and forty-eight hours [11].

Oluleye et al. [12] in describing the pattern of the presentation of chloroquine maculopathy in Ibadan, suggested that chloroquine should be withdrawn from the market and only be obtained with a doctor's prescription [12]. More so, with the COVID-19 pandemic, misinformed members of the public may resort to the use of hydroxychloroquine as a preventive measure against COVID-19 thereby predisposing themselves to grave harm.

\section{Conclusion}

There is no known proven prophylaxis or treatment against the toxicity of hydroxychloroquine on the eye, therefore a detailed eye examination following the recommended guidelines should be strictly adhered to in order to prevent irreversible blindness.

\section{Recommendations}

Health care workers should create awareness among the populace on irreversible loss of vision associated with the use of chloroquine and hydroxychloroquine. Patent medicine dealers should not sell hydroxychloroquine to patients except with a verified doctor's prescription.

\section{Acknowledgment}

The authors are grateful to the management of Lily hospital limited for providing a safe and conducive atmosphere for working and learning. Professor Macdonald Idu and Dr. Ayobami Chioma Egbu proofread this article, we appreciate their effort. We are grateful to Miss Onavwose Princess for providing technical assistance for this work.

\section{Patient Consent}

An informed consent was obtained from the patients and the study followed the tenets of the declaration of Helsinki and its amendments for research involving human subjects.

\section{References}

1. Nika M, Blachley T, Edwards P, Lee PP, Stein JD (2014) Are long-term chloroquine or hydroxychloroquine users being checked regularly for toxic maculopathy? JAMA Ophthalmol 132(10): 1199-1208.

2. Easterbrook M (1999) Detection and prevention of maculopathy associated with antimalarial agents. Int Ophthalmol Clin 39(2): 49-57.

3. Hansen MS, Schuman SG (2011) Hydroxychloroquine-induced retinal toxicity. Ophthalmic Pearls, USA. pp. 33-35.

4. Michaelides M, Stover NB, Francis PJ, Weleber RG (2011) Retinal toxicity associated with hydroxychloroquine and chloroquine: Risk factors, screening, and progression despite cessation of therapy. Arch Ophthalmol 129(1): 30-39.

5. Weiner A, Sandberg MA, Gaudio AR, Kini MM, Berson EL (1991) Hydroxychloroquine retinopathy. Am J Ophthalmol 112(5): 528-534.

6. Yam JCS, Kwok AKH (2006) Ocular toxicity of hydroxychloroquine. Hong Kong Med J 12(4): 294-304.

7. Schrezenmeier E, Dörner $T$ (2020) Mechanisms of action of hydroxychloroquine and chloroquine: Implications for rheumatology. Nat Rev Rheumatol 16(3):155-166.

8. Camichael AJ (1992) Hydroxychloroquine: A guide to usage. J Dermatolog Treat 3(2):103-106.

9. Yusuf IH, Sharma S, Luqmani R, Downes SM (2017) Hydroxychloroquine retinopathy. Eye 31(6): 828-845.

10. White NJ, Watson JA, Hoglund RM, Chan XHS, Cheah PY, et al. (2020) COVID-19 prevention and treatment: A critical analysis of chloroquine and hydroxychloroquine clinical pharmacology. PLoS Medicine 17(10): e1003252.

11. Khuroo MS (2020) Chloroquine and hydroxychloroquine in coronavirus disease 2019 (COVID-19). Facts, fiction and the hype: A critical appraisal. Int J Antimicrob Agents 56(3): 106101.

12. Oluleye TS, Babalola Y, Ijaduola M (2020) Chloroquine retinopathy: Pattern of presentation in Ibadan, Sub-Sahara Africa. Eye (Lond) 30(1): 64-67.

For possible submissions Click below: 